\title{
Quasi Moyamoya Disease: Case report and literature review
}

\author{
Srijana Neupane ${ }^{1}$, Karuna Tamrakar Karki ${ }^{1}$ \\ ${ }^{1}$ Department of Neurosurgery, B and C Medical College Teaching Hospital and Research Center, Birtamode, \\ Jhapa, Nepal \\ Correspondence: \\ Dr. Karuna Tamrakar Karki \\ Department of Neurosurgery \\ B and C Medical College Teaching Hospital and Research Center, Birtamode, Jhapa, Nepal \\ Email: tamrakarkaruna@gmail.com \\ Phone:+977-9843405124
}

\begin{abstract}
Quasi moyamoya disease is the unilateral form of typical moyamoya disease which is associated with acute or chronic stages of inflammatory diseases. Here, I present 21 -year-old boy presented with sudden onset of weakness of right sided limbs following physical workout, had acute ischemic infarction of left internal capsule in diffusion restriction MR image and unilateral steno- occlusive changes of terminal portion of left internal carotid artery was noted in digital subtraction angiography.
\end{abstract}

Key Words: hemiparesis, Ischemic stroke, moyamoya disease, young

$\mathrm{M}$ oyamoya Disease (MMD) was defined in 1969 by Suzuki and Takaku, ${ }^{1}$ is a progressive cerebrovascular disorder characterized by progressive stenosis of the terminal portion of the bilateral internal carotid arteries. The incidence of MMD peaks in two age groups; children who are approximately 5 years of age and adults in their mid-40s. There are nearly twice as many female patients as male patients. ${ }^{2}$ Although this disease is idiopathic, it is also sometimes associated with an underlying disease. With the association of underlying diseases, it is called quasi-moyamoya disease. ${ }^{3}$ Quasi MMD has been reported several times in English Literature with presence of unilateral angiographic findings of MMD associated with acute or chronic inflammatory conditions like atherosclerosis, autoimmune disease, type 1 neurofibromatosis, thyroid disease. ${ }^{4}$

\section{Case Description:}

39

Date submitted: 20/ 06/2021

Date accepted: 1/07/2021
A 21-years-old Mongolian boy with right hand predominance was brought to emergency department for right sided limb weakness and slurring of speech for one day. Weakness was sudden in onset following physical work out. There was no associated trauma, loss of consciousness, fever, night sweats, photophobia, neck stiffness or visual disturbances. There was a history of dull aching type of pain over left eye 3 days back which resolved within a day without any medications. He had no any significant past medical and surgical history. Physical examination revealed blood pressure-120/80 $\mathrm{mmHg}$, spo2-98\%, and respiratory rate-20 breaths per minute, pulse rate 86 per minutes with regular beats and temperature was 98.2 degree Fahrenheit. Glasgow Coma Scale of $15 / 15$ and bilateral pupillary size and reaction were normal. Motor power in left upper limb was $3 / 5$ and was recorded as $4 / 5$ in left lower limb. CT scan of head revealed ischemic changes in left internal capsular region. MRI brain demonstrated acute ischemic infarction over the posterior limb of left 
internal capsule (Figure 1A). Echocardiography and Doppler study of bilateral carotid artery revealed normal. Digital subtraction Angiography (DSA) lateral oblique view revealed small caliber internal carotid artery (ICA) with beading appearance. Steno-occlusive changes of terminal portion of left internal carotid artery with initial appearance of moyamoya vessels showing early "puff of smoke" was noted. (Figure 1B)

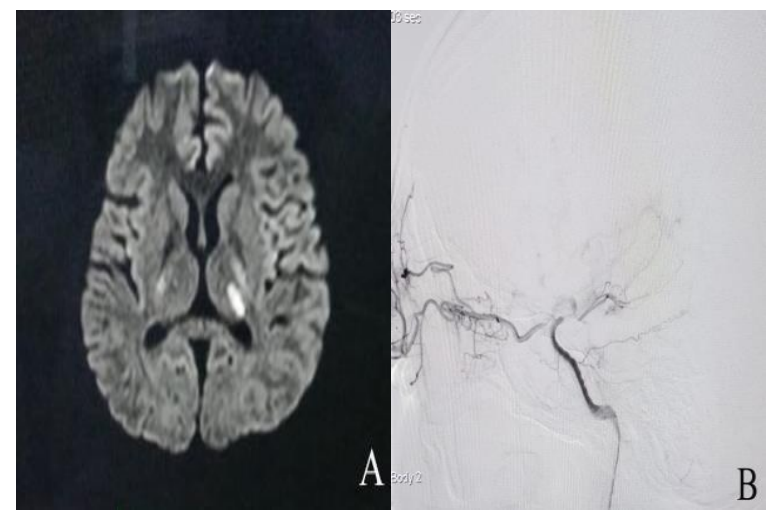

Figure 1: MR Diffusion restricted image showing restricted diffusion over posterior limb of left internal capsule suggesting acute ischemic infarction (A) and Digital subtraction angiography lateral oblique view demonstrating steno-occlusive changes of proximal ICA with early development of moyamoya vessel (puff of smoke) (B).

\section{Discussion}

Moyamoya disease is a cerebrovascular pathology, characterized by unilateral or bilateral stenosis or occlusion of intracranial segment of ICA and proximal parts of anterior and middle cerebral arteries. $^{5-7}$ Progressive stenosis and occlusion of intracranial internal carotid arteries occurs due to hypertrophy of smooth muscle in the vessel walls. ${ }^{8}$ The posterior cerebral artery is also involved in $\approx 30 \%$ of patients with moyamoya disease. ${ }^{9}$ The cerebral vasculature attempts to compensate by developing collateral circulation, and smaller blood vessels begin to enlarge, and hypertrophy becomes more visible on the diagnostic cerebral angiogram. The formation of collateral blood vessels gives the angiographic appearance of a "puff of smoke", which is roughly translated as "Moyamoya" in Japanese. $^{10}$

According to the laboratory research, presence of pro inflammatory cytokines in addition to mutation in RNF213 leads to increase process of angiogenesis and thickening of vascular wall. ${ }^{11}$ Another study had demonstrated association between increase levels of (Matrix Metalloproteinase) MMP-9, (Hepatocyte Growth Factor) HGF in CSF and MMD. These factors have potent role in induction of development of new vessels. Role of basic fibroblast growth factor bFGF and transforming growth factor beta-1 in mechanism of angiogenesis had also been documented in the Literature. ${ }^{12}$

An ischemic event is the commonest initial presentation of MMD which is more frequent in children than adults. Children commonly present with developmental delay, mental retardation and intractable seizure. These are the consequences of recurrent ischemic events due to progression of MMD. The progressive stenosis causes decreased cerebral blood flow to the brain parenchyma, causing ischemic and a compensatory vascular response. $^{3} \quad$ Subsequently, parenchymal, leptomeningeal and transdural collaterals develop in the ischemic brain as a response to hypoxia. ${ }^{8-10}$ Ischemic event can manifest as either transient ischemic attack or ischemic stroke. Certain factors like, excessive crying and hyperventilation can create sudden alteration on cerebral blood flow leads to cerebral hypoperfusion. ${ }^{4}$ Our reported case suddenly developed limb weakness and slurring of speech while doing physical exercise for British Army recruitment.

Deep lacunars, multiple dots, border zone and territorial involvement are the typical pattern of MMD whereas non territorial, honeycombing and gyral involvement are the atypical pattern of cerebral infarction reported in the Literature. ${ }^{13}$ Intracranial bleed is another frequent presentation of MMD that is common in adults than in children. Bleeding is commonly seen in anterior circulation than in other location. Headaches are also frequent in children with underlying MMD. Redistribution and alteration in cerebral blood flow might be the pathophysiology for recurrent headache in pediatric population. MMD presented with involuntary movement like chorea, focal dystonia had also been reported in Pediatric age group due to decrease blood flow in basal ganglia thalamo-cortical pathway. ${ }^{14}$ 
Conventional catheter angiography or DSA is a gold standard for the accurate diagnosis of MMD. Angiographic classification of MMD given by Suzuki elucidates about pathological changes rather than severity of MMD. Initial stage (early stage1-3) demonstrates occlusion of distal ICA with development of moyamoya vessels. ${ }^{15}$ (Early late stage 4-5) demonstrates anastomosis of ICA and external carotid artery (ECA) followed by vanishing of abnormal vessels. And in late stage 6 progressive vanishing of ICA is more demonstrated. Nonspecific ivy sign due to diffuse leptomeningeal enhancement may be noticeable in MR images. Increase visibility of deep medullary veins sometime gives the picture of brush like sign especially in susceptibility MR image. A high voltage slow wave on EEG is specific in pediatric age group due to acute ischemic event in MMD. ${ }^{11}$

\section{Conclusion:}

Criteria for moyamoya disease is bilateral involvement of internal carotid artery however due to increase finding of unilateral steno-occlusive changes seen in ICA in MMD, diagnostic criteria has been changed for MMD. Long standing or chronic inflammatory reaction that exists in suppressed state may be the cause of gradual progression of quasi MMD. They either present with acute ischemic event or intracranial hemorrhage. Particularly children may present with recurrent episodes of ischemia event or seizure.

\section{References:}

1. Suzuki J, Takaku A. Cerebrovascular "moyamoya" disease. Disease showing abnormal net-like vessels in base of brain. Arch Neurol. 1969 Mar; 20(3):288-99.

2. Scott RM, Smith ER. Moyamoya disease and moyamoya syndrome. N Engl J Med. 2009 Mar 19; 360(12):1226-37.

3. Mikami T, Suzuki H, Komatsu K, Mikuni N. Influence of Inflammatory Disease on the Pathophysiology of Moyamoya Disease and Quasi-moyamoya Disease. Neurol Med Chir (Tokyo). 2019 Oct 15; 59(10):361-370.

4. Kim JS. Moyamoya Disease: Epidemiology, Clinical Features, and Diagnosis. J Stroke. 2016 Jan;18(1):2-11

5. Handa J, Handa H: Progressive cerebral arterial occlusive disease: analysis of 27 cases. Neuroradiology 1972, 3(3):119-133.

6. Hasuo K, Tamura S, Kudo S, Uchino A, Carlos R, Matsushima T,Kurokawa T, Kitamura K, Matsuura K: Moyamoya disease: use of digital subtraction angiography in its diagnosis. Radiology 1985, 157(1):107-111.
7. Takahashi M: Magnification angiography in moyamoya disease: new observations on collateral vessels. Radiology 1980, 136(2):379-386.

8. Miyamoto S, Yoshimoto T, Hashimoto N, Okada Y, Tsuji I, Tominaga T, Nakagawara J, Takahashi JC; JAM Trial Investigators. Effects of extracranial-intracranial bypass for patients with hemorrhagic moyamoya disease: results of the Japan Adult Moyamoya Trial. Stroke. 2014 May;45(5):1415-21.

9. Kuroda S, Ishikawa T, Houkin K, Iwasaki Y. [Clinical significance of posterior cerebral artery stenosis/occlusion in moyamoya disease]. No Shinkei Geka. 2002 Dec; 30(12):1295-300.

10. Berry JA, Cortez V, Toor H, Saini H, Siddiqi J. Moyamoya: An Update and Review. Cureus. 2020 Oct 16;12(10):e10994.

11. Gupta A, Tyagi A, Romo M, Amoroso KC, Sonia F. Moyamoya Disease: A Review of Current Literature. Cureus. 2020 Aug 30;12(8):e10141

12. Malek AM, Connors S, Robertson RL, Folkman J, Scott RM. Elevation of cerebrospinal fluid levels of basic fibroblast growth factor in moyamoya and central nervous system disorders. Pediatr Neurosurg. 1997 Oct; 27(4):1829.

13. Cho HJ, Jung YH, Kim YD, Nam HS, Kim DS, Heo JH. The different infarct patterns between adulthood-onset and childhood-onset moyamoya disease. J Neurol Neurosurg Psychiatry. 2011 Jan; 82(1):38-40.

14. Baik JS, Lee MS. Movement disorders associated with moyamoya disease: a report of 4 new cases and a review of literatures. Mov Disord. 2010 Jul 30; 25(10):1482-6.

15. Chinchure SD, Pendharkar HS, Gupta AK, Bodhey N, Harsha KJ. Adult onset moyamoya disease: institutional experience. Neurol India. 2011 Sep-Oct; 59(5):733-8. 